\title{
Mélusine 34 (2014). Le surréalisme et les arts du spectacle. Dir. Sophie Bastien et Henri Béhar, 2014. 315 p.
}

Sophie Bastien et Henri Béhar, qui ont dirigé ce numéro de Mélusine, postulent que le surréalisme, largement étudié en littérature et dans les arts visuels, mérite des recherches supplémentaires dans d'autres domaines. En particulier, il est opportun, malgré le peu d'intérêt que portait André Breton au théâtre, d'examiner ce mouvement, ses précédents et son influence, dans les arts du spectacle. Le volume qu'ils ont composé, regroupe des contributions fort intéressantes et fort bien rédigées (articles, comptes rendus, documentaire) et une courte pièce inédite (1911) de l'écrivain et dramaturge Georges Ribemont-Dessaignes qu'un article (Gilles Losseroy) analyse.

Michel Corvin s'intéresse au travail d'abstraction dans l'œuvre d'Alfred Jarry, contraire à « la surenchère du vivant» qui anime le surréalisme, à l'omniprésence du corps dans les productions théâtrales, alors que les surréalistes privilégient le langage, et, bien dans la veine surréaliste cette fois, aux mises en scène de l'inconscient chez Roger Vitrac. Diana Vlasie examine le motif baroque du theatrum mundi dans le roman Détours (1924) du surréaliste René Crevel. Misao Harada argumente que, chez Breton, le théâtre exprimait le rêve, tandis que Dina Mantchéva porte son attention sur «le rapport scène-salle dans le drame surréaliste ». Caroline Barbier de Reuille se penche sur les «spectacles scéniques » de Salvador Dalí et sur la mise en scène de sa vie. L'article d'Ion Pop porte sur le théâtre de Gallu Naum, co-fondateur du Groupe surréaliste roumain en 1940, et celui de Charlène Conts sur la formule «je m'oralise » de son autre co-fondateur, Gherasim Luca. Béhar, qui contribue aussi en fin de volume un florilège de messages autographes signés dans les exemplaires de ses ouvrages que Breton destinait à ses correspondants, établit, à l'aide d'exemples tirés de la pièce Eleutheria (1947), que Samuel Beckett s'intéressait à l'esthétique surréaliste en dépit de ses réticences. Marie-Claude Hubert voit en Eugène Ionesco « un héritier du surréalisme », bien qu'il n'ait pas adhéré au mouvement, dans la mesure où il partageait les vues exprimées dans le premier Manifeste (1924) de Breton, à savoir l'éloge de l'imagination, de la folie et du freudisme. Selon Martine Antle, Marie Ndiaye, dans l'usage de motifs cultivés par les surréalistes, en serait une héritière d'une génération encore plus jeune. Noële Racine rappelle, pour sa part, que le «langage exploréen» du dramaturge Claude Gauvreau, automatiste montréalais, est le cousin «surrationnel» des pratiques surréalistes. Dans son analyse du roman La marge (1967), Gabriel Saad s'attache à 
définir «la fonction des arts du spectacle dans la poétique narrative» d'André Pieyre de Mandiargues. Jean-Yves Samacher identifie dans le théâtre d'Antonin Artaud des «propriétés performatives » et une pratique «d'ordre existentiel » pour les mettre en rapport avec la création contemporaine. Martin Mercier fournit une «rhétorique du geste théâtral d'inspiration surréaliste » qui privilégie, entre autres, l'ellipse, l'hypallage et l'apophonie. Laurence Tuot décèle dans le design culinaire des dernières années des affinités avec «les installations surréalistes du siècle passé », qu'il s'agisse, par exemple, de « sacs en pain, [de] viandes de potau-feu totémiques, [de] chimères de tous poils ou [d']écailles à dévorer». Finalement, Fabienne Douls Eicher étudie les «ruptures de sens » d'un poème de Pablo Picasso et Richard Spiteri cherche des antécédents baudelairiens dans l'œuvre poétique de Benjamin Péret. Elodie Gaden, Ionna Papaspyridou et Marc Kober rendent compte dans le détail d'ouvrages parus respectivement sur les avant-gardes, Jacques Prévert et Albert Cossery.

Le surréalisme et les arts du spectacle ouvre ainsi de nouvelles pistes dans les recherches surréalistes, élargit le champ d'investigation à un large répertoire et offre une scène fascinante des traces et dérivations surréalistes.

Pauline Brise 